\section{Note on Ion Exchange with Mercury(II)}

\section{ERIK HÖGFELDT and MAMOUN MUHAMMED}

Department of Inorganic Chemistry, Royal Institute of Technology, S.100 44 Stockholm 70, Sweden

L

ittle is known about the ion exchange behaviour of mercury ${ }^{1}$ in spite of the great interest in tracing its fate in polluted waters, etc. We have started an investigation of the ion exchange properties of $\mathrm{Hg}$ (II) using ion exchangers with various types of ion exchanging sites. Since this work has to be discontinued at present we think the results obtained so far may be of some general interest.

Batch experiments were performed at $25^{\circ} \mathrm{C}$, the ionic medium being $0.1 \mathrm{M} \mathrm{HNO}_{3}$. The concentration of $\mathrm{Hg}(\mathrm{II})$ varied between $10^{-2}$ and $10^{-5}$ M. Mercury was determined radiometrically as ${ }^{203} \mathrm{Hg}$ employing a Tracerlab Compumatic Scaler and a Tracerlab scintillation counter model Sc-57A for $\gamma$-rays. After equilibrium had been attained the two phases were separated by centrifugation and filtration through glass-wool. The filtrate was tested with a Tyndall lamp for colloids present. Only clear samples were accepted for analysis.

Table 1. Capacities (mequiv./g resin) of partially air dried ion exchange resins.

\section{Resin}

Capacity

Dowex 50W X-8

$3.76 \pm 0.01$

Dowex Al

Amberlite IRC-50

$4.49 \pm 0.05$

$6.96 \pm 0.1$

Sephadex SH

$0.17 \pm 0.01$

The resins used were Dowex 50W X-8, Dowex Al, Amberlite IRC-50, and Sephadex SH. Table 1 gives the capacities of the resins. In the case of Dowex 50 this was determined by emf-titration; in all other cases by back titration with acid after the addition of excess sodium hy. droxide solution to the acid form of the resin.

Fig. 1 gives $\log \varkappa$ against $\log \left[\mathrm{Hg}^{2+}\right]_{\mathrm{eq}}$ for the three resins: Dowex 50, Amberlite

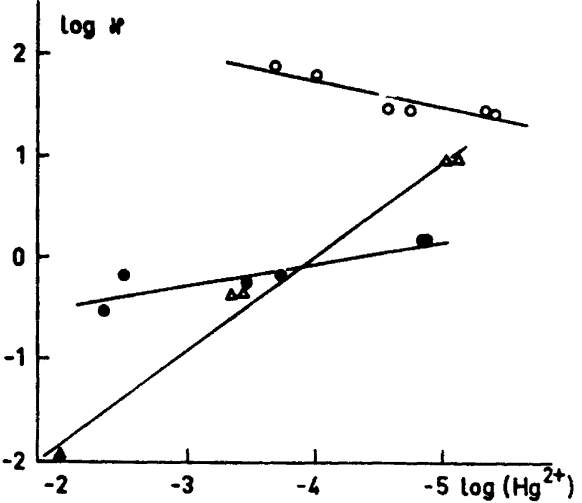

Fig. 1. The logarithm of the equilibrium quotient $(x)$ plotted against the logarithm of the equilibrium concentration of mercury $\left[\mathrm{Hg}^{2+}\right]$ in the aqueous phase. $O$ Dowex 50W X-8; Amberlite IRC-50; $\triangle$ Dowex Al.

IRC-50, and Dowex A1; $x$ is the equilibrium quotient for the reaction

$$
\begin{gathered}
\mathrm{Hg}^{2+}+2 \mathrm{HR} \rightleftharpoons \mathrm{HgR}_{2}+2 \mathrm{H}^{+} \\
x=\frac{X_{\mathrm{HgR}_{2}}}{X^{2} \mathrm{HR}} \cdot \frac{\left[\mathrm{H}^{+}\right]^{2}}{\left[\mathrm{Hg}^{2+}\right]}
\end{gathered}
$$

$X_{\mathrm{HgR}}$ is the equivalent fraction of $\mathrm{Hg}^{2+}$ in the resin. At the acidity used, Dowex 50 has a better affinity for $\mathrm{Hg}^{2}+$ than Amberlite IRC-50 and Dowex A1, which are both weak acid resins with a low "effective" capacity. It is interesting to note the very large change in affinity for mercury with Dowex Al. A possible explanation might be some impurity in the resin that exchanges at the low pH used. Elution experiments with $1 \mathrm{M} \mathrm{HNO}_{3}$ showed that at least $99 \%$ of the mercury was eluted. Reduction can thus hardly be the explanation of the large variation in $x$.

The results with Sephadex SH are not given in Fig. 1 because in this case the amount of mercury taken up by the resin always exceeded the experimentally determined capacity. A possible explanation is partial reduction of mercury by the resin, which is known to show electron exchange properties. $^{3}$

This work has been given financial support by Naturvårdsverket. Professor Torbjörn Westermark is gratefully acknowledged for supply. ing a sample of the Sephadex resin.

Acta Chem. Scand. 24 (1970) No. 7 
1. Heering, R. Chelatbildende Ionenaus tauschern, Akademieverlag, Berlin 1967.

2. Högfeldt, E. Arkiv Kemi 26 (1967) 325.

3. Jellum, E. Acta Chem. Scand. 18 (1964) 1887.

Received August 27, 1970.

\section{Preparation of Methyl-substituted Trithienylphosphines and Their Sulfides HANS JØRGEN JAKOBSEN}

Department of Organic Chemistry, University
of Aarhus, DK-8000 Aarhus C, Denmark

In connection with current studies of Inuclear magnetic resonance spectra of aromatic and heteroaromatic phosphine derivatives $^{1}$ it was of interest to obtain information concerning the signs and magnitudes of the expected four-, five-, and six-bond methyl proton-ring phosphorus long-range spin-spin coupling constants in phosphorus substituted methylthiophenes. In this paper the preparations of the six hitherto unknown isomeric tri-(methylthienyl)-phosphines (Ia-VIa) and their sulfides $(\mathrm{Ib}-\mathrm{VIb})$ are described.

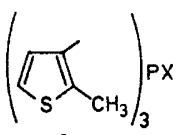

1

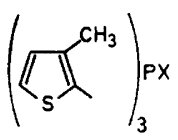

IV

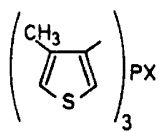

II

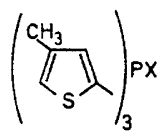

$\nabla$

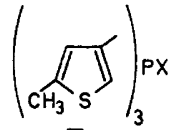

III

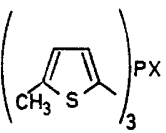

VI a: $\mathrm{X}=$ lone pair

b: $\mathrm{X}=\mathrm{S}$

The phosphines were prepared in $28-$ $66 \%$ yields from the corresponding methylthienyllithium derivatives, obtained either by low-temperature metal-halogen interconversion of the methylbromothiophenes (compounds Ia-IVa) or by direct metallation of the methylthiophenes (com- pounds Va and VIa) with butyllithium, followed by reaction with phosphorus tribromide. The phosphines were converted to their sulfides in quantitative yields by reaction with elementary sulfur in solutions of methanol or chloroform. Structures and purities of the isolated compounds were checked by ${ }^{1} \mathrm{H}$ NMR spectroscopy, mass spectrometry, or by microanalyses. All compounds were obtained in a pure state, with the exception of the mixture of 4 methyl-2-thienyllithium (ca. 83\%) and 3-methyl-2-thienyllithium (ca. $17 \%$ ) resulting from metallation of 3-methylthiophene with butyllithium ${ }^{2}$ which only allowed the isolation of tri-(4-methyl-2-thienyl)-phosphine (Va) as an oily mixture (ca. $54 \%$ ) with some of the isomers di-(4-methyl-2thienyl)-3-methyl-2-thienyl phosphine (ca. $40 \%)$ and di-(3-methyl-2-thienyl)-4methyl-2-thienyl phosphine (ca. $6 \%$ ) as deduced from ${ }^{1} \mathrm{H}$ NMR spectra. Separation by means of gas chromatography or thin layer chromatography could not be achieved. Attempts to prepare (Va) in a pure state by other synthetic routes were not carried out as the complete analysis of its ${ }^{1} \mathrm{H}$ NMR spectrum ${ }^{3}$ could be performed on the mixture of isomers. However, the corresponding sulfide (Vb), prepared from the isomeric mixture, could easily be obtained in a pure crystalline state by fractional recrystallization. In the preparations of the phosphines (Ia-IIIa) low.boiling products, later shown ${ }^{4}$ to result from a thiophene ring-opening reaction, were obtained.

Experimental. Boiling and melting points are uncorrected.

Starting materials. 2- and 3-Methylthiophene were commercially available (Schuchardt, Munich) and used without further purification. 2-Methyl-3-bromothiophene was prepared according to Gronowitz et al..$^{5}$ using dimethyl sulfate instead of methyl $p$-toluenesulfonate, b.p. $55^{\circ} / 9 \mathrm{mmHg}$ (b.p. $56-58^{\circ} / 10 \mathrm{mmHg}$ ), yield $88 \%(84 \%){ }^{5} 4$-Methyl-3-bromothiophene was obtained from 4-bromo-3-thienyllithium ${ }^{5}$ and dimethyl sulfate, b.p. $58-60^{\circ} / 8-9 \mathrm{mmHg}$ (b.p. $\left.181-184^{\circ} / 770 \mathrm{mmHg}\right),{ }^{5}$ yield $90 \%$. 2-Methyl-4-bromothiophene was obtained from 4-bromo-2-thienyllithium ${ }^{6}$ and dimethyl sulfate (at $-70^{\circ}$ ) as an isomer-pure product $(>98 \%)$ which is inconsistent with the observations of Gronowitz and Frostling; ${ }^{7}$ b.p. $58-59^{\circ} / 9 \mathrm{mmHg}$ (b.p. $61-62^{\circ} / 11 \mathrm{mmH.g}$ ), yield $81 \%$. 3-Methyl-2-bromothiophene was prepared by the method of Steinkopf and Jacob. $^{8}$ 